\title{
Introduction au numéro spécial sur les dispositifs de formation à l'enseignement des mathématiques
}

\author{
Caroline Lajoie • Frédérick Tempier
}

Published online: 31 May 2019

(C) Ontario Institute for Educat. Studies 2019

Le thème de la formation des enseignants dans la recherche francophone en didactique des mathématiques a une histoire différente d'une région du monde à l'autre. Le cas du Québec et de la France en est une bonne illustration. À l'Université du Québec à Montréal, par exemple, les travaux de recherche en didactique des mathématiques se sont articulés dès le début des années soixante-dix à des préoccupations de formation initiale et continue des enseignants (Bednarz, 2001, 2007). En France, c'est au début des années quatrevingt-dix « que l'enseignant va commencer à devenir objet de recherche dans différents travaux, en même temps qu'augmente l'implication des didacticiens dans la formation des maîtres » (Margolinas et PerrinGlorian, 1997, p.10) et c'est à ce moment aussi que des recherches portant sur des questions de formation commencent à émerger (citons par exemple Houdement et Kuzniak, 1996). Ces différences laissent entrevoir une variété d'approches, de questions, de perspectives théoriques, de méthodologies, pour aborder le thème de la formation des enseignants dans les travaux menés par les chercheurs francophones.

Pour ce numéro spécial entièrement en français, auquel ont contribué des chercheurs des deux côtés de l'Atlantique (canadiens, belges et français), nous avons fait le choix d'entrer par l'étude de dispositifs de formation à l'enseignement des mathématiques visant le développement professionnel des enseignants. Le terme « dispositif de formation » regroupe ici des dispositifs de différentes « tailles », allant de « micro », de l'ordre d'une séance ou moins et relevant d'une ou plusieurs tâches de formation, jusqu'à « macro », sur un temps long et relevant de la mise en œuvre organisée en une progression d'un ensemble de tâches de formation. Les dispositifs de formation à l'étude sont mis en œuvre en lien plus ou moins proche avec le « terrain » ou avec la recherche en didactique des mathématiques. Ils touchent des publics variés, soit des enseignants débutants (formation théorique ou pratique) ou expérimentés (formation continue), du primaire ou du secondaire, voire même du postsecondaire. Aussi, ils peuvent amener les formés à adopter, en fonction des tâches qui le sous-tendent, différentes postures épistémologiques, comme par exemple celles d'élève, d'enseignant ou d'étudiant universitaire (Deblois et Squalli, 2002).

\footnotetext{
C. Lajoie $(\bowtie)$

Groupe de recherche sur la formation à l'enseignement des mathématiques (GREFEM), Université du Québec à Montréal, Montréal, Québec, Canada

e-mail: lajoie.caroline@uqam.ca

F. Tempier

Laboratoire de didactique André Revuz (EA 4434), UA, UCP, UPD, UPEC, URN, Université de Cergy-Pontoise,

Cergy-Pontoise, France

e-mail: frederick.tempier@u-cergy.fr
} 
Cette entrée par les dispositifs de formation permet d'interroger différents aspects de la formation, notamment la place et les types de savoirs en jeu, soit les savoirs mathématiques, didactiques, pédagogiques ou professionnels. Les dispositifs de formation permettent aussi d'interroger l'activité des formés ainsi que celle des formateurs en relation entre autres avec ces différents types de savoirs.

L'étude de dispositifs de formation s'avère toutefois complexe. Cette complexité peut tenir au fait que, souvent, les chercheurs sont eux-mêmes impliqués dans la formation en tant que formateurs, ce qui rend plus difficile la mise à distance nécessaire au travail de recherche. Il apparaît également que l' « épaisseur » des dispositifs de formation complexifie leur analyse. Ceux-ci mêlent, en effet, de façon imbriquée, des questions liées à la formation elle-même à des questions d'enseignement et d'apprentissage des mathématiques - l'enjeu réel de la formation —, ce qui amène à mettre au jour et à interroger différentes « couches » ou « niveaux » d'analyse. Des allers-retours entre ces différents niveaux peuvent alors être pris en charge par le dispositif de formation, ou non, ce qui peut être un révélateur du lien plus ou moins proche entre le dispositif de formation et les questions d'enseignement et d'apprentissage. Une telle entrée est celle utilisée, par exemple, par la COPIRELEM (Mangiante et al., 2019) ou encore par René de Cotret et Passaro (2018) pour l'étude de dispositifs de formation.

À l'origine de ce numéro spécial s'est déroulé à Paris en juillet 2017, dans le cadre des rencontres internationales $d u$ réseau de recherche en éducation et en formation (RÉF), un symposium intitulé « Efficience et adaptabilité de dispositifs de formation en mathématiques pour le développement professionnel des futurs enseignants ». Pour l'occasion, les participants, regroupés préalablement en cinq équipes (la plupart composées de chercheurs provenant de différents pays), étaient invités à présenter un dispositif de formation et à l'interroger selon différentes dimensions en s'inspirant de l'une ou l'autre (ou même de plusieurs) des questions suivantes :

- Quels sont les choix qui pilotent la conception du dispositif de formation ? Quels sont les savoirs mathématiques, didactiques et professionnels visés par le dispositif?

- Quelles sont les caractéristiques du dispositif mettant en jeu des connaissances mathématiques, didactiques et professionnelles et comment est réalisée l'articulation entre ces connaissances ?

- Comment sont pris en compte les besoins, les connaissances et les pratiques professionnelles des enseignants dans le dispositif de formation?

- Quelle(s) mise(s) en œuvre possible(s) du dispositif? Quelles adaptations ?

- Quels sont les effets du dispositif sur le développement professionnel des enseignants ?

Au terme du symposium, trois équipes ont soumis un article pour ce numéro spécial et d'autres chercheurs ont été invités à soumettre un article de manière à couvrir une plus grande variété d'approches, de cadres, d'outils et perspectives. Le numéro spécial débute par des articles présentant des choix de formation plus généraux qui nous permettent ensuite de basculer vers l'étude de dispositifs plus ponctuels. Nous avons gardé pour la fin du numéro spécial les dispositifs impliquant les nouvelles technologies, emboîtant ainsi en quelque sorte un pas vers l'avenir.

Dans un article intitulé « Enjeux et modalités de formation pour les professeurs des écoles en didactique des mathématiques », Pascale Masselot et Denis Butlen s'appuient sur des résultats de recherche à propos de la formation des professeurs des écoles en France (6 à 15 ans) et des pratiques de ces professeurs pour dégager, en termes d'enjeux et de modalités, cinq grands principes pour la conception et l'analyse d'une formation didactique holistique des professeurs des écoles, soit une formation prenant en compte à la fois la complexité et la cohérence des pratiques. Puis, ils mettent en évidence quatre leviers susceptibles d'être utilisés par les formateurs et illustrent la mise en œuvre des cinq grands principes dégagés précédemment par des exemples de formations de publics différents (futurs professeurs des écoles en formation et professeurs débutants ou plus expérimentés exerçant en éducation prioritaire).

Dans leur article «Une dimension particulière de la réflexivité : "les mathématiques comme problème professionnel" à la base d'un dispositif de formation initiale en didactique des mathématiques », Kevin 
Balhan, Isaline Gerard, Giang Nguyen Ngan et Maggy Schneider décrivent un dispositif mis en place pour la formation initiale des enseignants des trois dernières années du secondaire en Belgique francophone (15 à 18 ans) à l'Université de Liège pour développer la réflexivité des enseignants. En appui sur la théorie anthropologique du didactique, ils montrent comment les mathématiques, leur épistémologie et la didactique peuvent constituer des leviers pour atteindre cet objectif. Les thèmes mathématiques retenus pour illustrer leurs propos sont ceux de l'analyse mathématique et de la géométrie.

Dans l'article intitulé « Faire vivre une formation à l'enseignement des mathématiques par résolution de problèmes : le cas du cours "Didactique des mathématiques II et Laboratoire" à l'Université du Québec à Montréal », Jérôme Proulx, par le biais d'un récit de formation, présente une initiative de formation pour des futurs enseignants de mathématiques du secondaire au Québec (12-17 ans). Au fil de la description de ce cours, qui s'accompagne d'exemples d'activités de résolution de problèmes, les fondements sont présentés de manière à donner accès au rationnel du formateur/concepteur. Ces fondements mettent en évidence le parallèle qui s'installe entre les activités d'enseignement par résolution de problèmes proposées dans le cours et les activités de formation à cet enseignement.

Dans un article intitulé " Méthodologie pour l'étude didactique d'un entretien formateur/professeur stagiaire en mathématiques dans le cadre d'une visite de classe », Adolphe Adihou, Patrick Gibel et Sylvie Blanquart-Henry s'intéressent au dispositif de visite de classe dans la formation initiale des enseignants du second degré (secondaire), et plus particulièrement à l'entretien faisant suite à l'observation d'une séance de mathématiques mise en œuvre par le stagiaire. Prenant appui sur la double approche didactique et ergonomique et la théorie de l'agir professionnel des enseignants, les auteurs élaborent une méthodologie pour analyser le déroulement d'un tel entretien et les « effets » de celui-ci sur la capacité du stagiaire à adopter une attitude réflexive. Ils mettent ensuite en œuvre cette méthodologie pour analyser l'entretien formateur-stagiaire ayant suivi l'observation d'une séance en classe de seconde en France (15-16 ans) dans le domaine des statistiques.

Dans un article intitulé « Former à aider un élève en mathématiques : une étude des potentialités d'un scénario de formation basé sur un jeu de rôles », Caroline Lajoie, Christine Mangiante, Pascale Masselot, Frédérick Tempier et Claire Winder Guille-Biel rendent compte d'une exportation dans le contexte de la formation initiale des professeurs des écoles en France (6 à 11 ans) d'un dispositif de formation initialement développé à l'Université du Québec à Montréal : le jeu de rôles. Le scénario de formation au cœur de cet article est basé sur un jeu de rôles plongeant les futurs enseignants dans une situation d'aide à un élève rencontrant une difficulté dans une tâche portant sur les nombres décimaux. L'analyse préalable du scénario de formation s'appuie sur une modélisation théorique des différents niveaux de tâches impliquées. Les connaissances et pratiques ayant émergé dans le contexte de la mise en œuvre effective de ce scénario auprès de deux groupes d'étudiants en formation initiale en France sont documentées, et des potentialités du dispositif pour faire évoluer ces connaissances et pratiques sont dégagées.

Dans l'article intitulé « Les simulateurs informatiques, ressources pour la formation des enseignants de mathématiques », le dispositif analysé est un simulateur informatique de pratiques d'enseignement des mathématiques. Fabien Emprin et Hussein Sabra y définissent trois grands critères généraux à prendre en compte dans la conception d'un tel simulateur, en s'appuyant, d'une part, sur les bénéfices et limites liés à l'introduction des technologies numériques dans la formation des enseignants, et, d'autre part, sur deux cadres théoriques permettant de définir, comprendre et analyser les pratiques enseignantes ayant recours aux technologies, soit la double approche didactique et ergonomique et l'approche instrumentale. Les auteurs montrent ensuite comment ils ont eux-mêmes pris en compte ces critères dans la conception du simulateur qui plonge l'utilisateur dans une classe où les élèves doivent résoudre un problème ouvert en géométrie avec l'aide d'un logiciel de géométrie dynamique.

Dans le dernier article de ce numéro spécial, intitulé « Conception de tâches MERLO : un dispositif dans la formation continue pour l'enseignement de la géométrie », Mathieu Thibault et Nathalie Sinclair présentent les résultats d'une recherche exploratoire impliquant la conception de tâches MERLO par des enseignants du secondaire (12-18 ans) engagés dans un processus de formation continue à l'Université 
Simon Fraser (Colombie Britannique, Canada). Dans le cadre de cette recherche, la conception de tâches MERLO est mise en place pour permettre à des enseignants de décortiquer des concepts géométriques du secondaire. À partir d'exemples de tâches élaborées par les enseignants, les auteurs dégagent des enjeux importants que le dispositif permet d'éclairer, en particulier en lien avec la coordination des registres de représentation visuel et langagier. L'incorporation de figures dynamiques dans la conception de tâches amène par ailleurs les auteurs à proposer d'élargir la théorie de Raymond Duval en proposant un nouveau registre de représentation, soit le registre dynamique.

Nous espérons que ce numéro spécial sera le début d'une réflexion approfondie, appuyée sur la recherche, sur les dispositifs de formation à l'enseignement des mathématiques, et qu'il incitera d'autres chercheurs du Canada et d'ailleurs dans le monde à nourrir cette réflexion.

\section{Références}

Bednarz, N. (2001). Didactique des mathématiques et formation des enseignants: le cas de l'Université du Québec à Montréal. Revue canadienne d'enseignement des mathématiques, sciences et technologies, 1(1), 61-80.

Bednarz, N. (2007). Ancrage de la didactique des mathématiques au Québec: à la recherche de sens et de cohérence. Dans Actes du colloque du Groupe de Didactique des Mathématiques au Québec (pp. 21-61).

DeBlois, L. et Squalli, H. (2002). Implication de l'analyse de productions d'élèves dans la formation des maitres du primaire. Educational Studies in Mathematics, 50(2), 212-237.

Houdement, C. et Kuzniak, A. (1996). Autour des stratégies utilisées pour former les maîtres du premier degré en mathématiques. Recherches en didactique des mathématiques, 16(3), 289-322.

Mangiante, C., Masselot, P., Petitfour, E., Simard, A., Tempier, F., Winder, C. (2019). Pratiques de formation en mathématiques des professeurs des écoles : un cadre pour analyser les potentialités de situations de formation. Dans I. Verscheure, M. Ducrey-Monnier, et L. Pelissier (dir.), Enseignement et formation : éclairages de la didactique comparée. Toulouse, France : Presses Universitaires du Midi.

Margolinas, C. et Perrin-Glorian, M.-J. (1997). Des recherches visant à modéliser le rôle de l'enseignant (éditorial du volume 17/3). Recherches en Didactique des Mathématiques, 17/3, 7-16.

René de Cotret, S. et Passaro, V. (2018). « J'avais jamais vu ça comme ça avant ! Un projet de recherche-action collaborative pour la formation continue en mathématiques au secondaire. Dans J.-L. Dorier et S. Coutat (dir.) Actes du colloque Espace Mathématique Francophone EMF 2018 (SPE4, pp. 20-21).

Publisher's Note Springer Nature remains neutral with regard to jurisdictional claims in published maps and institutional affiliations. 